\title{
X-ray and Neutron Reflectivity
}

\author{
Moshe Deutsch \\ Physics Department, Bar Ilan University, Ramat Gan 52900, Israel \\ B. M. Ocko
}

Physics Department, Brookhaven National Laboratory, Upton, NY 11973, U.S.A.

October 4, 1994

\section{Contents}

1 Introduction 2

2 Theory of Reflectivity 4

2.1 General Features . . . . . . . . . . . . . . . . . . . 4

2.2 Dynamical theory of reflectivity $\ldots \ldots \ldots \ldots \ldots \ldots \ldots$

2.3 Kinematical theory of reflectivity $\ldots \ldots \ldots \ldots \ldots \ldots \ldots \ldots \ldots \ldots \ldots \ldots$

2.4 Unique features of neutron reflectivity $\ldots \ldots \ldots \ldots \ldots \ldots \ldots$

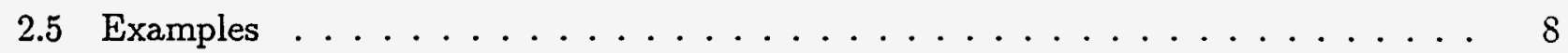

2.5 .1 Rough surfaces $\ldots \ldots \ldots \ldots \ldots \ldots \ldots$

2.5 .2 A thin film on a substrate $\ldots \ldots \ldots \ldots \ldots \ldots$

2.5 .3 Crystal surface $\ldots \ldots \ldots \ldots \ldots \ldots \ldots \ldots \ldots \ldots \ldots \ldots \ldots \ldots$

$\begin{array}{lll}3 \text { Instrumentation. } & 10\end{array}$

4 Applications 11

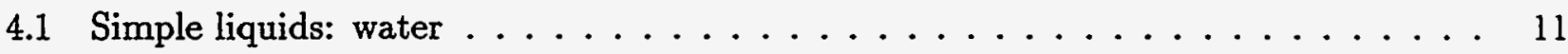

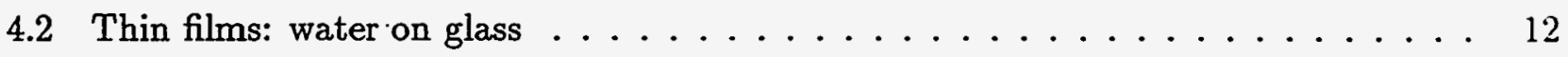

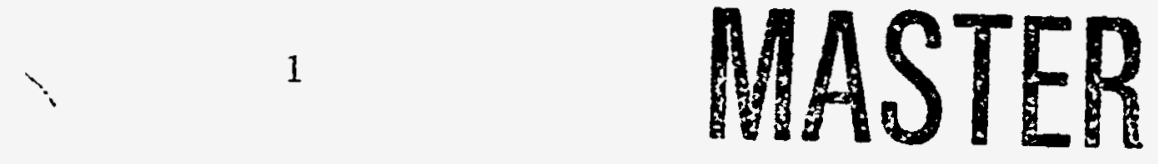

DISTRIBUTION OF. THIS DOCUMENT IS UNLIMITED DT 
4.3 Polymer films: combined X-ray and neutron reflectivity . . . . . . . . . 13

4.4 Polarized neutron reflectivity: ultra thin Fe films . . . . . . . . . . . . 13

4.5 Crystalline surfaces: clean $\mathrm{Au}(001)$ in vacuum $\ldots \ldots \ldots \ldots \ldots \ldots$

5 Related techniques $\quad \mathbf{1 5}$

$\begin{array}{lll}6 & \text { List of works cited } & 19\end{array}$

7 Further reading $\quad 20$

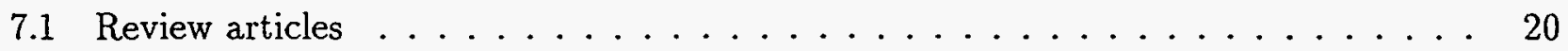

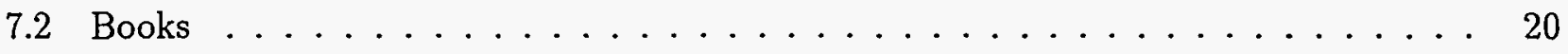

\section{Introduction}

The intensity of X-rays and neutrons reflected from an interface at near-grazing incidence depends sensitively on the refractive index profile normal to the interface. As the refractive index depends on the density, the measured reflectivity vs. incidence angle can be used to determine the interfacial density profile with sub-Ångstrom resolution. The density sampled is, however, different for the two probes. As neutrons interact with the atomic nuclei, they probe the scattering amplitude density profile of the nuclei. This amounts effectively to probing the average mass density profile, where the contribution of each atomic species is weighted by its nucleus-neutron interaction strength, which varies irregularly throughout the periodic table. X-rays, however, interact with the electronic charge and thus probe the total electronic density profile of the interface. The two probes provide, therefore, slightly different, though related, information on the interface structure. Since modern synchrotron X-ray sources are 5-6 orders of magnitude brighter than the best neutron sources, X-ray reflectivity measurements routinely achieve much higher resolutions and accuracies than their neutron counterparts. However, the magnetic interaction and the large variation of the neutron scattering length upon isotopic substitution renders neutron reflectivity a method-of-choice in special cases such as studies of surface magnetism and the adsorption of polymers at liquid and

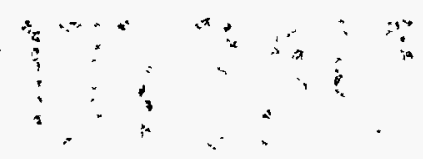




\section{DISCLAIMER}

This report was prepared as an account of work sponsored by an agency of the United States Government. Neither the United States Government nor any agency thereof, nor any of their employees, make any warranty, express or implied, or assumes any legal liability or responsibility for the accuracy, completeness, or usefulness of any information, apparatus, product, or process disclosed, or represents that its use would not infringe privately owned rights. Reference herein to any specific commercial product, process, or service by trade name, trademark, manufacturer, or otherwise does not necessarily constitute or imply its endorsement, recommendation, or favoring by the United States Government or any agency thereof. The views and opinions of authors expressed herein do not necessarily state or reflect those of the United States Government or any agency thereof. 


\section{DISCLAIMER}

Portions of this document may be illegible in electronic image products. Images are produced from the best available original document. 
solid surfaces.

The use of X-ray reflectivity to measure the index of refraction of matter was originally suggested by Compton (1923). Parratt (1954) revived this method and used it in his seminal study of glass-deposited thin metal films. Marra, Eisenberger, and Cho (1979) first applied the very small penetration depth for low angles of incidence to study the surface structure of crystals, opening up a new and very active field of research, which has provided a wealth of new knowledge about surface reconstruction and quasi-2D phase transitions. The $\mathrm{X}$-ray reflectivity studies of the free surfaces of liquid metals (Lu and Rice, 1978) and of liquid crystals (Als-Nielsen et al., 1982, Pershan and Als-Nielsen, 1984) laid the foundations for the fast-growing field of surface studies of liquids and soft condensed matter. These methods provided true microscopic information on important phenomena like surface freezing and melting and the structure of the various phases of monomolecular Langmuir films. These techniques were later extended to solid-solid crystalline, liquid-solid, and even liquid-liquid interfaces.

The first detailed theoretical treatment of X-ray reflectivity was given by Picht (1929). Parratt (1954) provided the first dynamical treatment, based on ideas drawn from classical optics of multilayers. The distorted-wave Born approximation theory of Vineyard (1982) was an important step beyond the kinematical theory (Born approximation). Since then a large number of theoretical studies of both specular reflection and the accompanying diffuse scattering have been published. taking into account an increasing number of real effects like surface roughness, capillary surface waves in liquids, static and dynamic disorder in solid surfaces and the stepped nature of crystalline surfaces.

Neutron reflectivity was first employed by Fermi (1946) to measure the scattering length and phase shift of the neutron upon reflection. Hughes and Burgy (1951) pioneered the use of neutron reflectivity to study surface magnetism, a method further developed by Felcher (1981) and coworkers. More recently, Penfold and Thomas (1990) studied polymer adsorption at, and surfactant monolayers on the free surface of mixtures of $\mathrm{D}_{2} \mathrm{O}$ and $\mathrm{H}_{2} \mathrm{O}$ by ingeniously using the isotope variation of the scattering length to eliminate the signal originating from the water. Surfaces of solid polymers and interfaces between polymeric bulks were also studied extensively by Russell (1990) 
and others.

The fast and wide dissemination of X-ray reflectivity and related surface methods since the 1980's is mainly due to two important factors. The first is the advent of powerful synchrotron radiation sources, dedicated to the production of X-rays. They provide intense, highly collimated X-ray beams which are prerequisites for these grazing angle surface studies. The second is the explosive growth in computer power since the 1970's, which put powerful computer fitting, modeling and simulation methods within easy reach of small groups and individual researchers. A similar, though more modest growth rate was enjoyed by neutron reflectivity. This growth was largely due to the construction of high power research reactors and spallation sources in the U.S.A, U.K. and France. With the development of brighter thermal neutron sources a faster increase is expected in the use of neutron reflectivity for surface studies.

\section{Theory of Reflectivity}

\subsection{General Features}

Standard electromagnetic theory (Born and Wolf, 1975) predicts the splitting of a plane wave incident on an interface between two media of refractive indices $n_{1} \neq n_{2}$ into reflected and refracted waves, as shown in Fig.1a. While $\alpha_{i}=a_{r}$ regardless of $n_{1}, n_{2}$, the angle of refraction $a^{\prime}$ is determined by Snell's law:

$$
n_{1} \cos \alpha_{i}=n_{2} \cos \alpha^{\prime}
$$

For both $\mathrm{X}$-rays and neutrons the complex refractive index $n$ is given by:

$$
n=1-\delta+\mathrm{i} \beta
$$

For X-rays,

$$
\delta=r_{0} \rho_{e} \lambda^{2} / 2 \pi, \quad \beta=\mu \lambda / 4 \pi
$$

where $r_{0}$ is the classical electron radius, $\lambda$ is the wavelength, $\rho_{e}$ is the electron density and $\mu$ is the mass absorption coefficient. For neutrons $r_{0} \rho_{e}$ is replaced by the neutron scattering length density, 
b. The weak interactions render $\delta$ in Eq. (2) small, of order $10^{-5}-10^{-6}$ for all materials. The absorption term $\beta<<\delta$ in most cases and, for simplicity, will be neglected from now on.

Free surfaces of solids and liquids are by far the most studied interfaces. Here $n_{1}=1$, and since for X-rays $\delta>0, n=n_{2}<1$. Snell's law yields $\alpha^{\prime}<\alpha_{i}$ and thus, upon decreasing $\alpha_{i}$, a finite angle $\alpha_{c}=\sqrt{2 \delta}$ is reached where $\alpha^{\prime}=0$. For an angle of incidence smaller than this critical angle total external reflection of the incident wave occurs, i.e., the reflectance, defined as $r=\mathbf{E}_{r} / \mathbf{E}_{i}$, is unity for $\alpha_{i}<\alpha_{c}$ and so is, of course, the reflectivity $R=|\mathrm{r}|^{2}$.

For angles $\alpha_{i}>\alpha_{c}, \mathbf{r}$ and $R$ are no longer unity, and for an infinitely sharp and flat surface, are given by Fresnel's law:

$$
r=|\mathrm{r}|=\left(q-q^{\prime}\right) /\left(q+q^{\prime}\right) \quad \text { and } \quad R_{F}(q)=\left|\left(q-q^{\prime}\right) /\left(q+q^{\prime}\right)\right|^{2} \text {, }
$$

where $q=(4 \pi / \lambda) \sin \alpha_{i}$ is the momentum transfer perpendicular to the surface, $q^{\prime}=\sqrt{q^{2}-q_{c}^{2}}$ and the absorption is assumed to be negligible. The reflectivity, $R$, is a fast decreasing function, and for $q \gg q_{c}$ is rery closely approximated by $R_{F}(q) \approx\left(q_{c} / 2 q\right)^{4}$. In the field of small angle bulk scattering this is long known as Porod's law. In practice, critical angles are a few tenths of a degree, and reflectivities at $\sim 20 q_{c}$, just a few degrees, are of order $\sim 10^{-\tau}$. Thus. the well collimated and very intense X-ray beams available at synchrotron sources, are the tools of choice for reflectivity measurements. Note that because of the low angles of incidence the reflectivity is practically polarization independent.

Real interfaces are neither abrupt nor ideally flat. On an atomic scale the transition between adjacent media is of a finite width. For example, thermally induced capillary waves in liquids, and structural defects or crystalline surface steps in solids modify the density profile. The reflectivity off a real surface will therefore deviate from Fresnel's law. Two main approaches exist for calculating the reflectivity in these cases. The first is the dynamical approach originally proposed by Parrat. The second is the kinematical approach, based on the first Born approximation. While the first of these is more rigorous, the second is much easier to use in practice and yields in the vast majority of cases accurate and reliable density profiles. We will now discuss each of these methods separately. 


\subsection{Dynamical theory of reflectivity}

The density variation perpendicular to the interface can be approximated by a piecewise-constant function, thus replacing the actual interface by a series of $N$ slabs having each a constant refractive index $n_{j}=1-\delta_{j}$ and a width $d_{j}$. By increasing the number and decreasing the width of the slabs, the approximation can be made arbitrarily close to the actual density profile. The first slab, $j=1$, denotes the medium above the free surface (e.g the vacuum), and the last, $N$-th, slab is the bulk where $d_{N+1}=\infty$. Consider now the $j$-th slab, depicted in Fig. $1 \mathrm{~b}$. The continuity requirements of the tangential components of the field at each interface yield for the reflectance $r_{j}$ of slab $j$ :

$$
r_{j}=a_{j-1}^{4}\left(r_{j+1}+F_{j}\right) /\left(r_{j+1} F_{j}+1\right)
$$

where $a_{j}=\exp \left(-\mathrm{i} q_{j} d_{j} / 4\right)$ and $F_{j}=\left(q_{j-1}^{\prime}-q_{j}^{\prime}\right) /\left(q_{j-1}^{\prime}+q_{j}^{\prime}\right)$ and $q_{j}^{\prime}=\sqrt{q^{2}-q_{c, j}^{2}}$ with $q_{c, j}$ denoting the critical angle for slab $j$. Eq. (5) is solved iteratively, by noting that since $d_{N}=\infty$ the reflectance off the bottom of the $N$-th slab is zero, and the sole contribution of that slab is from the upper interface. This yields $r_{N}=a_{N-1}^{4} F_{N}$, which is then used to calculate $r_{N-1}$ and so on until the free surface reflectance $r_{2}$ is obtain, whence $R(q)=r_{2}^{2}$. This method properly accounts for the effects of refraction and is therefore exact. Absorption can also be conveniently included by using a complex momentum tranisfer $q_{j}^{\prime}=\sqrt{q^{2}-q_{c, j}^{2}+\mathrm{i} \mu_{j} / 2}$. An additional advantage of the dynamical theory is that it gives a natural description of the many important cases involving layered interfaces such as ordered diblock polymers, thin films on a solid substrate or monomolecular Langmuir films on water. For more than a few layers, however, its use becomes very tedious analytically, although the iterative algorithm is very easy to program for computer calculations. By contrast, the approximate kinematical theory, given below, has the advantage of yielding a closed form expression for the reflectivity, which is sufficiently accurate for most cases.

\subsection{Kinematical theory of reflectivity}

Consider again the multislab approximation discussed above. This time, however, we assume small reflectivities and neglect refraction effects so that each slab reflects the incident ware independently. 
The amplitude ratio of the reflected-to-incident wave for a single slab $j$ at depth $z$ below the nominal surface is given by :

$$
E_{r}^{j} / E_{i}=4 \pi \mathrm{i} r_{0}<\rho_{e}(z)>d_{j} / q
$$

where $\langle\cdots\rangle$ denotes averaging in the $x-y$ plane, parallel to the surface. Making the slabs of equal infinitesimal thickness $\mathrm{d} z$, and summing the contributions over $z$, multiplying each by the appropriate phase factor $\exp (\mathrm{i} q z)$ one obtains the so called "master equation":

$$
R(q)=R_{F}(q)\left|\left(1 / \rho_{\infty}\right) \int_{-\infty}^{\infty}\left(\mathrm{d}<\rho_{e}(z)>/ \mathrm{d} z\right) \exp (\mathrm{i} q z) \mathrm{d} z\right|^{2}
$$

where $\rho_{\infty}$ is the bulk electron density. Thus, the Fresnel reflectivity is modified by the absolute value of the Fourier transform of the density gradient, which is, within a constant, the standard crystallographic structure factor of the interface. Conservatively, the master equation applies for $q \geq 4 q_{c}$, but it is often accurate enough right down to the critical angle. It has the advantage of being easily applicable to analytic models of the density profile and is therefore by far the most widely used expression for analyzing reflectivity measurements. It should be noted, however, that since Eq. (7) employs the square of the structure factor the phase information is lost just as in standard crýstallography, and a unique inversion of the measured $R(q)$ to obtain $\rho_{e}(z)$ is not possible in the general case. However, through judicious use of additional information and general physical constraints it is possible in almost all cases to obtain a well defined and accurate description of the density profile.

\subsection{Unique features of neutron reflectivity}

Neutrons have several unique properties which can be used to advantage in particular cases. For some materials (e.g. silicon) the scattering and absorption cross sections are extremely small, thus neutrons can travel through these materials with little attenuation. This feature can be used to advantage when studying thin organic films in contact with liquid interfaces. Secondly, for $\mathrm{x}$-rays the scattering length density always has the same sign, whereas for neutrons $\delta$ can assume both negative and positive values for different atoms and isotopes. In particular, $H$ and $D$ have $b$ values 
of opposite signs. Hence an appropriate mixture of $\mathrm{D}_{2} \mathrm{O}$ and $\mathrm{H}_{2} \mathrm{O}$ can be prepared ( molar ratio of 0.088:1 ) such that its average scattering length is equal to that of air. The free surface of such a mixture will not reflect or refract an incident neutron beam and the general concept is referred to as contrast matching. The cancellation of the bulk signal greatly improves the signal/noise ratio in experiments involving monolayers on water, surface adsorption of polymers in solution and solute concentration near a free surface of water. Furthermore, by deuterating a specific part of an organic or biomolecule one can study by neutron reflection the behaviour of that part only near the surface. Although anomalous dispersion of $\mathrm{X}$-rays near an absorption edge was also employed for similar measurements, neutrons are superior to X-rays in this respect.

Another advantage of neutrons are their magnetic interaction with matter. For magnetic materials $b=b_{0} \pm C \mu$ where $\mu$ is the magnetic moment density and the sign depends on whether the neutron polarization is parallel $(+)$ or antiparallel $(-)$ to the magnetization. Thus, polarized neutron reflectivity permits studies of magnetic structures near the surface. The measurement of the so called flipping ratio, the ratio of the parallel-to-antiparallel reflectivities, is particularly advantageous since the interference from many non-magnetic variables cancels out.

\subsection{Examples}

Fig. 2 shows a few typical examples of surface profiles and the resulting reflectivity curves. We now discuss each separately.

\subsubsection{Rough surfaces}

An important case, shown in Fig. 2a, is the "rough" surface, where the density profile perpendicular to the surface has a finite width. This may be the free surface of a liquid, broadened by thermally excited capillary waves, an amorphous solid surface with a random height distribution or a crystal surface with extra units cells missing or piled up at random positions on the crystallographic plane comprising the nominal "surface". Averaged over the $x-y$ plane, the perpendicular density profile of such a diffuse interface is well represented in many important cases by an error function. The 
master equation yields in this case:

$$
R(q) / R_{F}(q)=\exp \left(-q^{2} \sigma^{2}\right)
$$

where $\sigma$ is the width parameter of the height distribution. This Debye.Waller like term is used to represent diffuseness or roughness in profiles of more complicated structure. It is easily recognized by plotting $R / R_{F}$ vs. $q^{2}$ on a semilog scale, yielding a straight line, where the slope is $\sigma^{2}$. An example of this is shown in the inset in Fig. 3.

\subsubsection{A thin film on a substrate}

The reflectivity from a thin film of thickness $d$ and a uniform electron density $\rho_{e}$ on a substrate with $\rho_{e}^{\prime} \neq \rho_{e}$ and an equal roughness $\sigma$ at the upper and lower interfaces is shown in Fig. 2b. The master equation yields :

$$
R(q) / R(q)_{F}=\left[a^{2}+b^{2}+2 a b \cos (q d)\right] \exp \left(-q^{2} \sigma^{2}\right)
$$

where $b=\rho_{e} / \rho_{e}^{\prime}$ and $a=1-b$. The typical oscillatory reflectivity curve. often called Kiessig fringes, is the signature of such a film. The film thickness can be determined immediately from the curve by $d \approx 2 \pi /\left(q_{i}-q_{i+1}\right)$ where $q_{i}, q_{i+1}$ are the positions of two consecutive extrema.

\subsubsection{Crystal surface}

Assume a cubic crystal, having an abrupt surface parallel to a set of lattice planes $00 l$ of spacing $d_{00 l}$. Summation of the contributions from all planes in the kinematical approximation yields:

$$
R(q) \sim\left[1 / 4 q^{2} \sin ^{2}\left(q d_{00 l}\right)\right]^{2} \exp \left(-q^{2} \sigma_{b}^{2}\right)
$$

where $\sigma_{b}$ is the bulk Debye-Waller factor. For small $q, \sin (q) \sim q$ and Eq. (10) assumes the right $q^{-4}$ form of Fresnel's law. For $q=q_{00 l}=2 \pi l / d_{00 l}$, however, Bragg peaks are obtained, as shown in Fig. 2c. The "wings" of these peaks fall off as $\sim\left|\left(q-q_{00 l}\right)\right|^{-2}$. This shape is, of course, modified in real crystals by the molecular form factor, surface roughness, faceting, reconstruction and adsorption of foreign molecules at the surface. 


\section{Instrumentation.}

The spatial resolution in reflectivity measurements is inversely proportional to the angular range measured. High resolution entails therefore measuring out to large angles of incidence and consequently very low reflectivities. The use of high intensity X-ray beams available at synchrotron sources is thus very beneficial for many of these measurements. Present day (ca. 1994) second generation synchrotron sources and the associated beam line optics are capable of producing monochromatic fluxes of the order of $10^{11}$ photons per second in a $\leq 1 \mathrm{~mm}^{2}$ spot. This represents a flux increase of $\sim 10^{4}$ over conventional rotating-anode laboratory-based sources.

For solid samples, a conventional two-circle diffractometer can be used to measure the reflectivity. The sample is oriented in this case perpendicular to the rotation plane of the circles (usually the horizontal) and the reflectivity is measured in a $\theta-2 \theta$ scan as in a single crystal Bragg reflection measurement. Due to the small angle of incidence, the illuminated area ("footprint") on the sample is magnified greatly as compared to the beam width. For sufficiently large samples a narrow slit is positioned close to the sample to keep the footprint smaller than the size of the sample at all

angles of incidence. For samples that can not be made larger than the footprint, an angle-dependent illuminated area correction is required. Since measurements of grazing incidence diffraction. diffuse scattering etc. are usually carried out along with the reflectivity, four- or six-circle diffractometers are often emploved for these measurements, sometimes in conjunction with ultra-high vacuum environmental chambers to keep the surface of the samples atomically clean (Feidenhans'l, 1989 ; Robinson and Tweet, 1992).

Liquid samples dictate a different geometry. By virtue of their horizontal surfaces, the plane of reflection is always vertical. The incident beam must be, therefore, bent down to impinge on the surface at the required angle of incidence. As the beams generated at synchrotron sources are highly collimated and horizontal, the beam is reflected downward by a tilted crystal. At laboratory-based rotating anode sources, which produce a highly divergent beam, the incident beam is selected by a collimator looking back at the source at the required downgoing angle. In both cases, the sample is translated in the up-down direction as the angle of incidence is varied, to intercept the downgoing 
beam. Finally, the reflected beam is detected by a scintillator/photo-tube combination, an ion chamber, a diode, or a one or two dimensional detector.

Neutron reflectivity measurements are carried out at both thermal neutron reactors and spallation sources. At monochromatic thermal neutron sources, the reflectometers function similar to $\mathrm{X}$-ray reflectometers since Bragg optics can be used for neutrons as well as X-rays. Such reflectometers have been built in either the standard or liquid configurations. Reflectometers at spallation sources have been developed to take advantage of the relationship between the neutron wavelength and velocity (Felcher et. al, 1987). The sample and detector are fixed to provided the desired incident and exit angles $\alpha$ to satisfy the specular condition. The incident beam contains a spread of wavelengths with a known spectrum. The reflected beam is analyzed by a neutron time-of-flight technique to provide the reflected intensity for each wavelength. Through the relation $q=(4 \pi) / \lambda \sin \alpha$ this provides the required $R(q)$ curve. An important advantage of this energy dispersive mode of measurement is that the reflectivity is acquired for a range of $q$ values simultaneously without moving the sample, the incidence and exit slits and with a fixed area illuminated on the sample. Although similar energy dispersive X-ray reflectivity measurements hare been done, they are more complicated experimentally and seldom used in practice.

Several current (ca. 1994) reflectometers are described in detail by Russell (1990):

\section{Applications}

\subsection{Simple liquids: water}

The liquid-vapour interface is intrinsically very sharp and flat. Its reflectivity should therefore closely follow the Fresnel Law $R_{F}(q)$. The water/vapour interface is perhaps the most common interface in nature and one of the first studied with X-ray reflectivity. The measured reflectivity (Braslau et. al, 1988) is reproduced in Fig. 3 along with the Fresnel Law. Below $q_{c}=(4 \pi / \lambda) \sin \alpha_{c}=0.0217 \AA^{-1}$, the reflectivity is nearly unity as expected. In the range $q_{c} \leq q \leq 0.4 \AA^{-1}$ the reflectivity decreases by over seven orders of magnitude. While at low $q$ values the measured curve is seen to follow 
closely Fresnel's law (solid line), as expected, above $q=0.15 \AA^{-1}$, the data deviates incrcasingly from Fresnel's Law. This deviation is exceptionally well described by the Gaussian function given in Eq.(8) as apparent from the inset to Fig 3. The best fit to the data yields an interfacial profile width of $\sigma=3.3 \pm 0.1 \AA$. This was shown to be predominantly due to thermally induced capillary waves in addition to a small intrinsic interfacial width. The dominant capillary contribution is predicted to have a width $\sim 2.8 \AA$, in excellent agreement with the measurements. The capillary wave model also predicts the dependence of the width upon the spectrometer resolution, surface tension and temperature. All of threse effects have been confirmed experimentally. Measurements of the diffuse scattering close to the specular direction also support the capillary wave model. For liquid metals, the surface tensions of which are much higher than that of water, the surface roughness should be dominated by the intrinsic interface width, of order $1 \AA$.

\subsection{Thin films: water on glass}

For thin films on a substrate, the waves reflected from the upper and lower interfaces of the film interfere and yield a modulated reflectivity curve. The X-ray reflectivity curve of a thin water layer on a smooth borasilicate float glass substrate is shown in Fig. + (Garoff et. al, 1989). The periodic oscillations in the curve are apparent. The monotonic decrease of their amplitude with $q$ is due to the effects of roughness. The period of $\lrcorner q=0.069 \AA^{-1}$ corresponds to a water layer thickness of $d=(2 \pi) / \Delta q=91 \AA$. The solid line shows a model reflectivity curve where the density profile incorporates a water/vapour interfacial width of $4.2 \AA$, an average water layer thickness of $91 \AA$, and a water/substrate interface width of $3 \AA$.

In addition to their scientific interest, thin films also have important industrial applications in the fields of coatings, electronics, biotechnology and medicine. X-ray reflectivity has become a powerful probe of the structure of these films and many films and multilayers were studied including semiconductors, metals and alloys, polymers, Langmuir-Blodgett films, Langmuir films on water, liquid crystals, and liquid helium. 


\subsection{Polymer films: combined X-ray and neutron reflectivity}

The study of block copolymer films on solid substrates, using both X-ray and neutron reflectivity, demonstrates the complementary nature of these two techniques. In Fig. 5, X-ray and neutron reflectivity data (Mayes et. al, 1994) is shown for a diblock copolymer film, cast onto a flat polished silicon substrate and annealed for 24 hours. The polymer consists of a deuterated polystyrene block (d-PS) and a normal polymethyl methacrylate block (PMMA). The PS, having a lower surface energy, aggregates upon annealing at the surface, while the PMMA resides at the silicon surface. As the electron density of the two components is similar, the internal layered structure of the film does not give rise to optical density variations for X-rays. Thus only the fringes due to the total thickness of the film are observed in the X-ray data. A constant density model fit to the X-ray data, shown in Fig. 5 as a solid line, shows very good agreement and yields a uniform film thickness of $\sim 1200 \AA$. By contrast, the different neutron scattering lengths of hydrogen and deuterium, the first being positive and the other negative, result in very different optical densities for the internal layers for neutrons. This gives rise to additional interference beyond that reflecting the film's total thickness, as can be seen in the neutron data in Fig. 5 . A model fit to the data, shown in a solid line, yields a thickness of $\sim 290 \AA$ for each of the PM and PMMA layers, with a diffuse transition layer of $50 \AA$ between them and a surface roughness of $6 \AA$. As can be seen, the agreement between the model and data is excellent.

A large number of similar studies on different polymers have been carried out providing a wealth of information about the statics and kinetics of phase separation in polymer blends and block copolymers.

\subsection{Polarized neutron reflectivity: ultra thin Fe films}

Theoretical studies indicate that magnetic moments of atoms at the surface of metals should be greater than those of bulk atoms. The same should hold for atoms in ultra-thin metallic films. Polarized neutron reflectivity has been successfully implemented to study this and related issues in a number of metallic films. 
One such study (Huang, Liu, and Felcher, 1993) addressed ultra-thin Fe films, ranging in thickness from 4 to $16 \AA$. The reflectivity from a $16 \AA$ film in a 200 Oe magnetic field at room temperature is shown in Fig. 6. As discussed above (Section 2.5.1), for neutron polarization parallel to the field, the usual nuclear scattering amplitude of the Fe atom is enhanced by the magnetization term $c B$, where $c$ is the magnetic moment and $B$ is the magnetic induction in the layer, generated by an assembly of aligned atomic magnetic moments $\mu$. For the other polarization, the scattering amplitude is decreased by the term $-c B$. The difference between the parallel (filled circles) and antiparallel (open circles) polarizations is apparent in the figure. By fitting the reflectivities for

both polarizations to a simple model the effective iron layer thickness, $14 \AA$, the iron scattering density, $7.0 \times 10^{-6} \AA^{-2}$, and the internal magnetic field, $B=4.0 \times 10^{-6} \AA$ have been obtained. These values indicates a (not-unreasonable) $13 \%$ decrease in the density of Fe in the film as compared to the bulk. Contrary to theoretical predictions, the magnetic moment per atom is determined to be $(2 \pm 0.2) \mu_{B}$, the same as that of bulk Fe, regardless of thickness. However, temperature dependent measurements show that the thinnest films exhibit a lower Curie temperature than that of the bulk and a magnetization axis tilted out of the film surface.

\subsection{Crystalline surfaces: clean $\mathrm{Au}(001)$ in vacuum}

For crystalline surfaces. X-ray reflectivity prorides information on the atomic arrangement of the topmost layers. As an example, the reflectivity from the Au(001) surface in UHV is shown in Fig. $T$ (Gibbs et. al, 1988). Note that the maximum $q$ measured, $\sim 5 \AA^{-1}$, is nearly an order of magnitude greater than what is typically achieved for simple liquids or thin films. For $\mathrm{Au}(001)$, the sample size limits the $q$ range to values greater than $q_{c}$. The Bragg peak at $q=3.08 \AA^{-1}$ corresponds to the bulk (002) reflection. The calculated reflectivity for an ideally terminated gold surface (dashed curve) deviates from the measured reflectivity, especially where the reflectivity is weak. This deviation results from the top gold layer which does not have the same symmetry and gold density as the underlying gold layers. Rather, the top gold layer is "reconstructed" i.e. the atoms are arranged in a hexagonal pattern with a density $25 \%$ greater than the underlying layers and the topmost interlayer 
spacing is $20 \%$ larger than the bulk (001) layer spacing of $2.04 \AA$. The predicted reflectivity for this model (solid line) describes the essential features of the data. The prominent asymmetry around the (002) peak results from the $20 \%$ lattice expansion. Above $1170 \mathrm{~K}$ the reconstruction vanishes. A similar reconstruction was observed by X-ray reflectivity under electrochemical conditions (Ocko et. al, 1990). Studies have been carried out on other crystalline facets, different metals, semiconductor surfaces, and at the electrode/electrolyte interface.

\section{Related techniques}

There are several important techniques, related to reflectivity, whence additional information on the structure of the surface can be derived. For example, the surface "roughness" reduces the specularly reflected intensity by scattering intensity out of the specular direction. This diffuse scattering is dominated by, and can be analyzed to yield information on, the nature of the in-plane correlations of the "roughness", and their range and spatial dependence (Sinha et. al, 1988).

A few techniques take advantage of the refracted beam. which, for angles of incidence smaller than the critical angle, becomes eranescent. It travels along the surface and has an amplitude which decays exponentially with depth below the surface. ts the penetration depth is of order $\sim 50 \AA$, the diffraction of this wave by the surface layer provides information on the structure of the surface layer, free from any obscuring signal from the bulk. This effect is the basis for the grazing incidence diffraction (GID) technique, a powerful tool for surface structure determination. Although it has been used to determine the liquid surface structure factor of liquid metals and alloys it is used most extensively for studying ordered surfaces and monolayers. Here the order in the surface translates into Bragg peaks, often unique to the surface and non-existent for the bulk. However, perpendicular to the surface the order is restricted: a single molecular layer for Langmuir films or a crystalline half-space for the free surface of a crystal. Thus, the intensity distribution in the GID peaks is not point-like as in a bulk Bragg peak, rather it is elongated perpendicular to the surface. These surface normal scattering distributions, often called Bragg Rods, are the two-dimensional equivalents of the Laue spots obtained in diffraction from bulk crystals, where the order is three-dimensional. The 
intensity distribution along the rod can be analyzed to yield information mostly on the structure of the scattering medium perpendicular to the surface. Note that unlike specular reflectivity, which yields similar information but averaged over all of the surface, the Bragg rods originate in the ordered regions and hence yield information on these regions only. This is particularly useful for studies of partially ordered surfaces and monolayers.

The variation of the penetration depth of the refracted beam with the angle of incidence has also been used to investigate the concentration dependence of a specific atom on depth below the surface. This is done by using radiation of a fixed wavelength above the absorption edge of the atom and measuring the intensity variation with the angle of incidence of the fluorescence from that atom. A related technique, called SEXAFS for Surface Extended Absorption Fine Structure, uses the intensity variation with incident wavelength of the fluorescence, at wavelengths close to, and above an absorption edge. Since the angle of incidence is kept below the critical for all wavelengths, the fluorescence originates in the surface layer only, due to the short penetration depth. This technique is useful for investigating the local structure near the given atom up to its second or third coordination sphere. 
Figure 1. (a) Reflection and refraction diagram at a free surface. Note that for X-rays the refractive index of matter is less than unity. Hence, the refraction angle $\alpha^{\prime}$ is smaller than the incidence or reflection ones, $\alpha_{i}$ and $\alpha_{r}$. (b) The various waves reflected and refracted at the lower and upper surfaces of one layer, $j$, in a multilayer structure. $n_{j}$ and $d_{j}$ are the layer's refractive index and thickness, respectively.

Figure 2. Prototypical reflectivity curves $R$ divided by the Fresnel Law $R_{F}$ (left) and their corresponding surface density profiles (right). The curves are discussed in Section 2.5.

Figure 3. X-ray reflectivity from the water/vapour interface (solid dots). The deviation. between the data and the Fresnel Law (solid line) is due to capillary-waves-dominated surface roughness. The inset shows that the Gaussian roughness model in Eq.(8) fits the measured reflectivity well with a roughness width parameter $\sigma=3.3 \pm 0.1 \AA$. Adapted from Braslau(1988). For further details see Section 4.1.

Figure 4. X-ray reflectivity from a $91 \AA$ thick water layer on a glass substrate. The oscillations in the reflectivity curve originate from the interference between rays reflected from the water/vapour and the glass/water interfaces. Adapted from Garoff (1989). For further details see Section 4.2 .

Figure 5. X-ray and neutron reflectivities from an annealed deuterated-hydrogenated di-block copolymer film cast onto on a silicon surface. The irregular oscillations in the neutron reflectivity result from interference due to the alternating deuterated-hydrogenated internal layered structure of the film, and the large variation of the neutron scattering length upon isotopic substitution. By contrast, since the electronic charge density is equal for both types of layers, no internal structure is observed in the $\mathrm{x}$ - ray reflectivity and only the regular fringes due to the total thickness of the film, $\sim 1200 \AA$, are observed. Adapted from Mayes (1993). Further details are given in Section 4.3.

Figure 6. Spin-dependent neutron reflectivity from a $16 \AA$ thick Fe [reproduced from film. A 200 Oe magnetic field aligns the spins parallel to the surface and transverse to the beam. Filled and open circles indicate data for neutron spin parallel $(+)$ and antiparallel $(-)$ to the applied field, respectively. Note the strong dependence of the measured curves on polarization. The inset describes the neutron potential for + and - spin neutrons going through the sample. Reproduced 
from Huang (1993) with permission of the American Physical Society. See Section 4.4 for further details.

Figure 7. X-ray reflectivity from the (001) surface of a single crystal of gold in ultra high vacuum. The Bragg peak at $q=3.08 \AA^{-1}$ is the bulk (002) reflection. The measured reflectivity (open circles) deviates from the one calculated for an ideally terminated gold surface (dashed curve), but is well described by a surface model (see Section 4.5 for details) where the top gold layer is reconstructed in an hexagonal structure different from that of the bulk (solid curve). Adapted from Gibbs (1988). 


\section{List of works cited}

Als-Nielsen, J., Christensen, F., Pershan, P.S. (1982), Phys Rev. Lett., 48, 1107-1110

Born, M., Wolf, E. (1975), Principles of Optics, Oxford: Pergamon.

Braslau, A., Pershan, P. S., Swislow, G., Ocko, B. M., Als-Nielsen, J. (1988), Phys Rev. A, 38, $2457-2470$.

Compton, A. H. (1923), Phil. Mag. 45. , 45, 1121-1131.

Felcher, G. P., (1981), Phys. Rev. B, 24, 1595-1598.

Felcher, G. P., Hilleke, R. O., Crawford, R.K., Haumann, J., Kleb, R., Ostrowski, G. (1987), Rev. Sci Inst., 58, 609-619.

Fermi, E., Zinn, W. (1946), Phys. Rev. , 70, 103-103.

Garoff, S., Sirota, E.B., Sinha, S.K., Stanley, H.B. (1989), J. Chem. Phys, 90, 7505-7514.

Gibbs, D., Ocko, B. M., Zehner, D. M., Mochrie, S. G. J. (1988), Phys Rev. B, 38, 7303-7310.

Huang, Y. Y., Liu, C., Felcher, G. P. (1993), Phys. Rev. B , 47, 183-189.

Hughes, D. J., Burgy, M. T. (1951) Phys. Rev., 81, 498-506.

Lu, B.C, Rice S.A. (1978), J. Chem. Phys, 68, 5558-5567.

Marra, W.C., Eisenberger. P.. Cho. A. Y. (1979). J. App. Phys.. 50, 6927-6933.

Mayes, A. M., Russell, T. P.. Bassereau, P., Baker, S. M.. Smith. G. S. (1994), Macromolecules, 27, 749-755.

Ocko, B.M., Wang, J., Davenport. A., Isaacs, H. (1990) Phys. Rev. Lett. , 65, 1466-1469.

Parratt, L.G. (1954), Phys. Rev., 95, 359-369.

Penfold, J., Thomas, R. K. (1990), J. Phys. Condens. Matter, 2, 1369-1412.

Pershan, P.S., Als-Nielsen, J. (1984), Phys Rev. Lett., 52, 759-762.

Picht, J. (1929) Ann. Phys. , 5, 433-496

Russell, T.P. (1990), Mater. Sci. Rep., 5, 171-271.

Sinha, S.K., Sirota, E.B., Garoff, S., Stanley, H.B. (1988), Phys. Rev. B, 38, 2297-2311.

Vineyard, G. (1982), Phys. Rev. B, 26, 4146-4159. 


\section{$7 \quad$ Further reading}

\subsection{Review articles}

Als-Nielsen, J., Kjær, K. (1989), in : T. Riste and D. Sherrington (Eds.), Phase Transitions in Soft Condensed Matter, New-York:Plenum. p. 113.

Als-Nielsen, J. (1991), in: G. S. Brown and D. E. Moncton (Eds.), Handbook on Synchrotron Radiation, Amsterdam:North-Holland. p. 471.

Feidenhans'l, R. (1989), Surf. Sci. Reports 10, 105-188.

Hamley, I.W., Pedersen, J.S (1994), J. Appl. Crystall. 27, 29-35. ; ibid. 36-49.

Penfold, J., Thomas, R. K. (1990), J. Phys. Condens. Matter, 2, 1369-1412.

Robinson, I.K., Tweet, D.J. (1992), Rep. Prog. Phys. 55, 599-651.

Russell, T.P. (1990), Mater. Sci. Rep., 5, 171-271.

Schlossman, M. L., Pershan, P. S. (1990) in: D. Langevin (Ed.), Light Scattering by Liquid Surfaces and Complementary Techniques, New-York:Dekker,p. 365-404.

\subsection{Books}

Bienfait, M., Gay J.M. (Eds.) (1990), Proc. First Internat. Conf. Surface X-ray and Neutron Scattering, in: J. Phys. (France) Colloq. C7

Dosch, H. (1992), Critical Phenomena at Surfaces and Interfaces, Berlin:Springer.

Felcher, G.P., Russell, T.P. (1991), Methods of Analysis and Interpretaion of Neutron Reflectivity Data, in: Physica B 173.

Zabel,H., Robinson, I. K. (Eds.) (1992), Surface X-ray and Neutron Scattering, Springer Proceedings in Physics, Vol. 61. Heidelberg:Springer. 


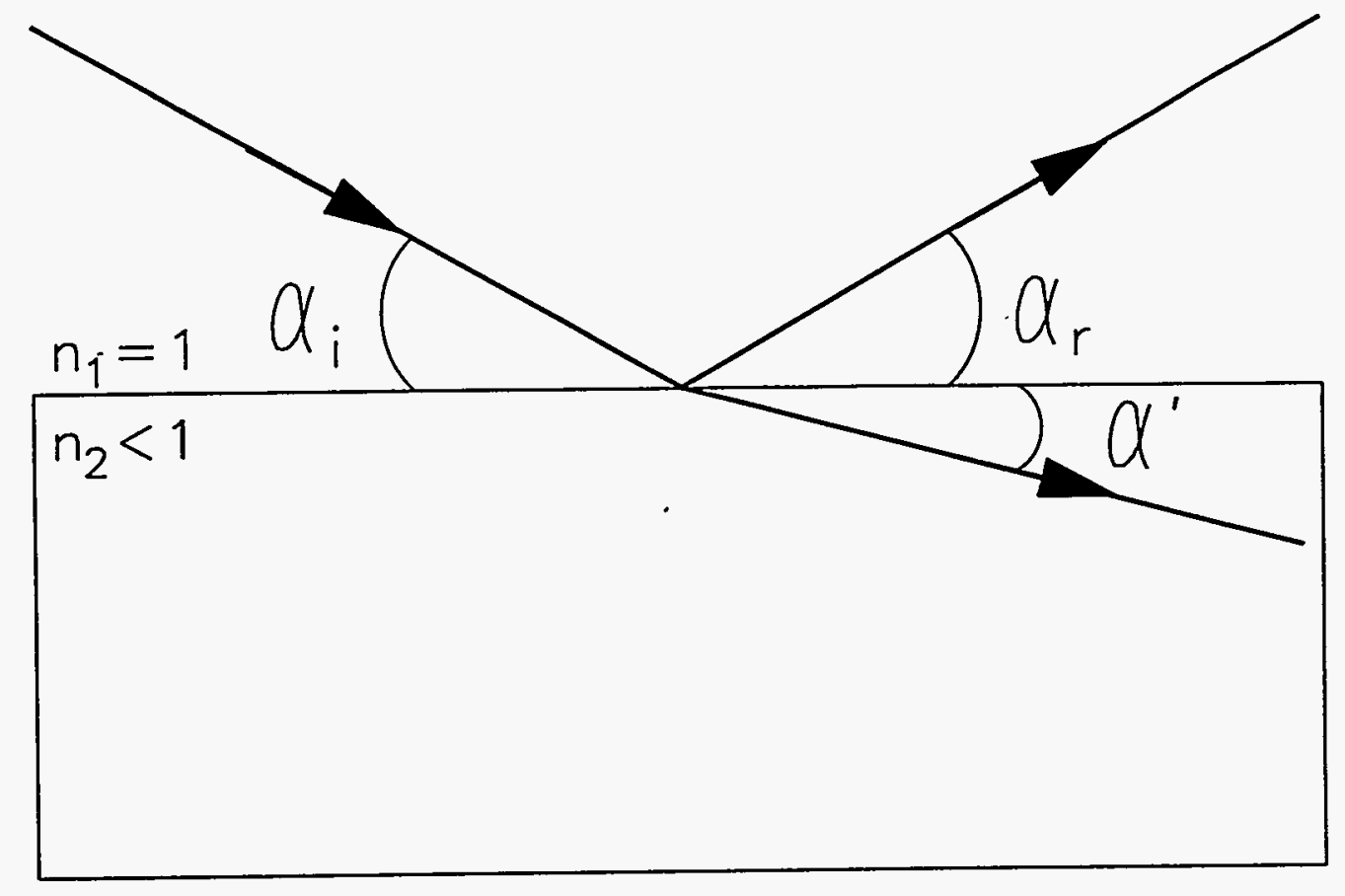

(b)

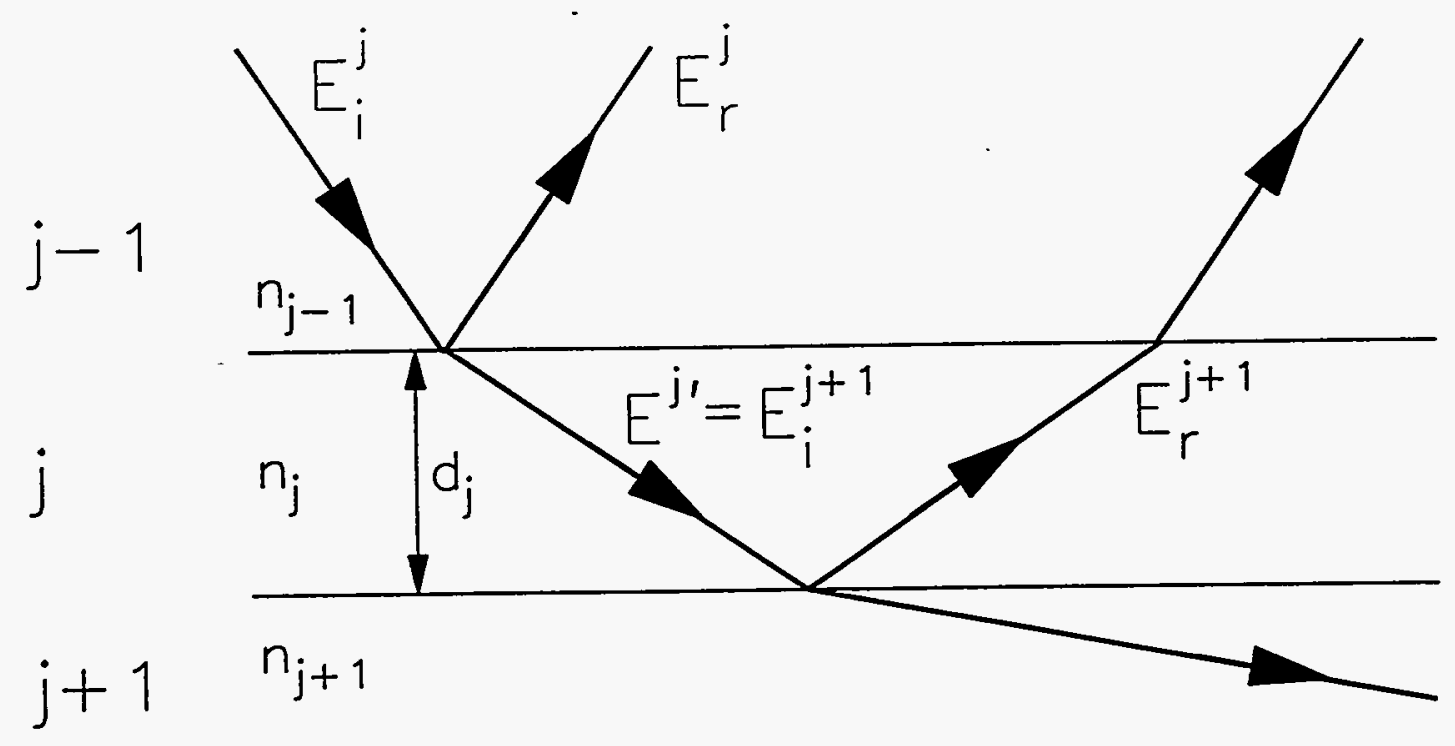

Fig. 1

Fig. 1 

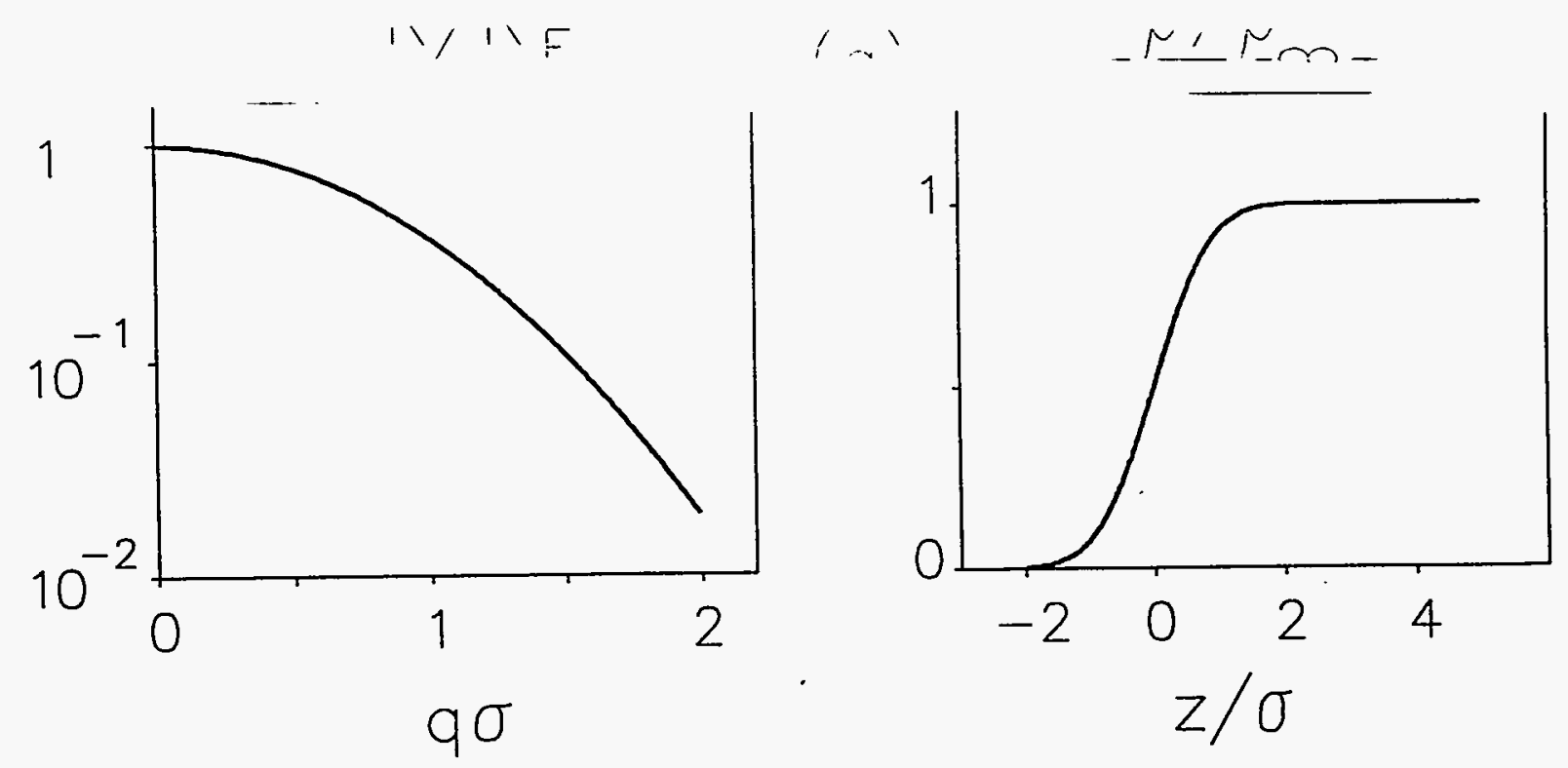

(b)
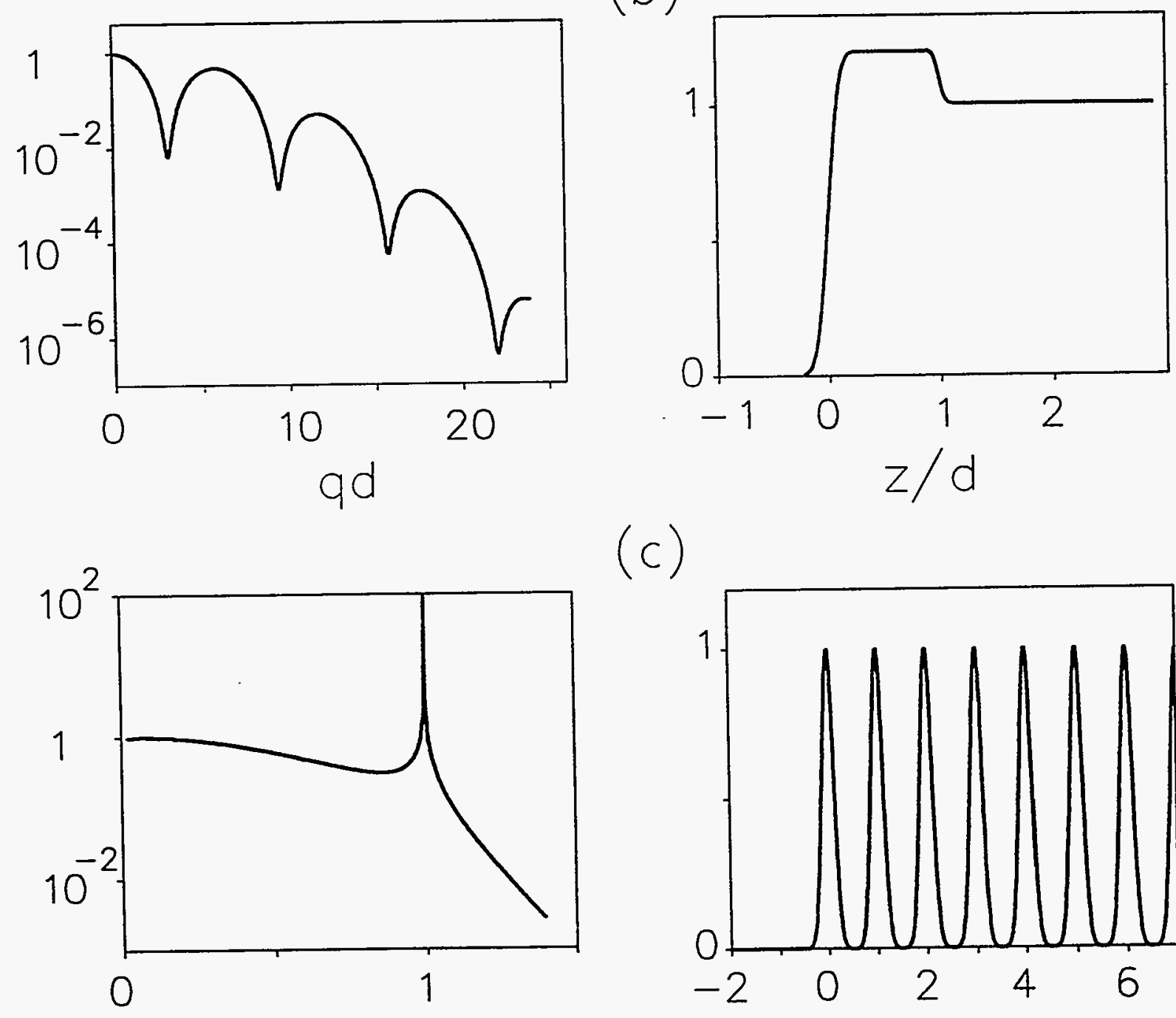

(c)

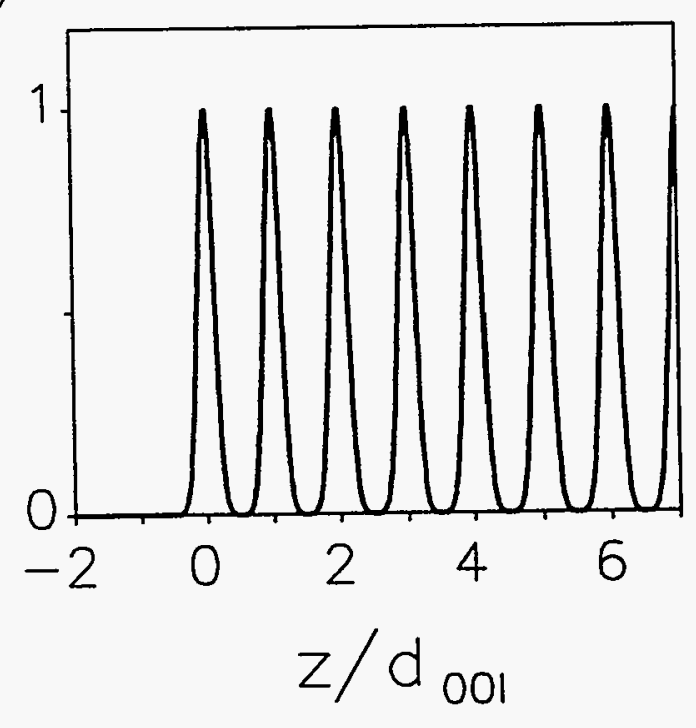

Fig. 2

Fig. 2 


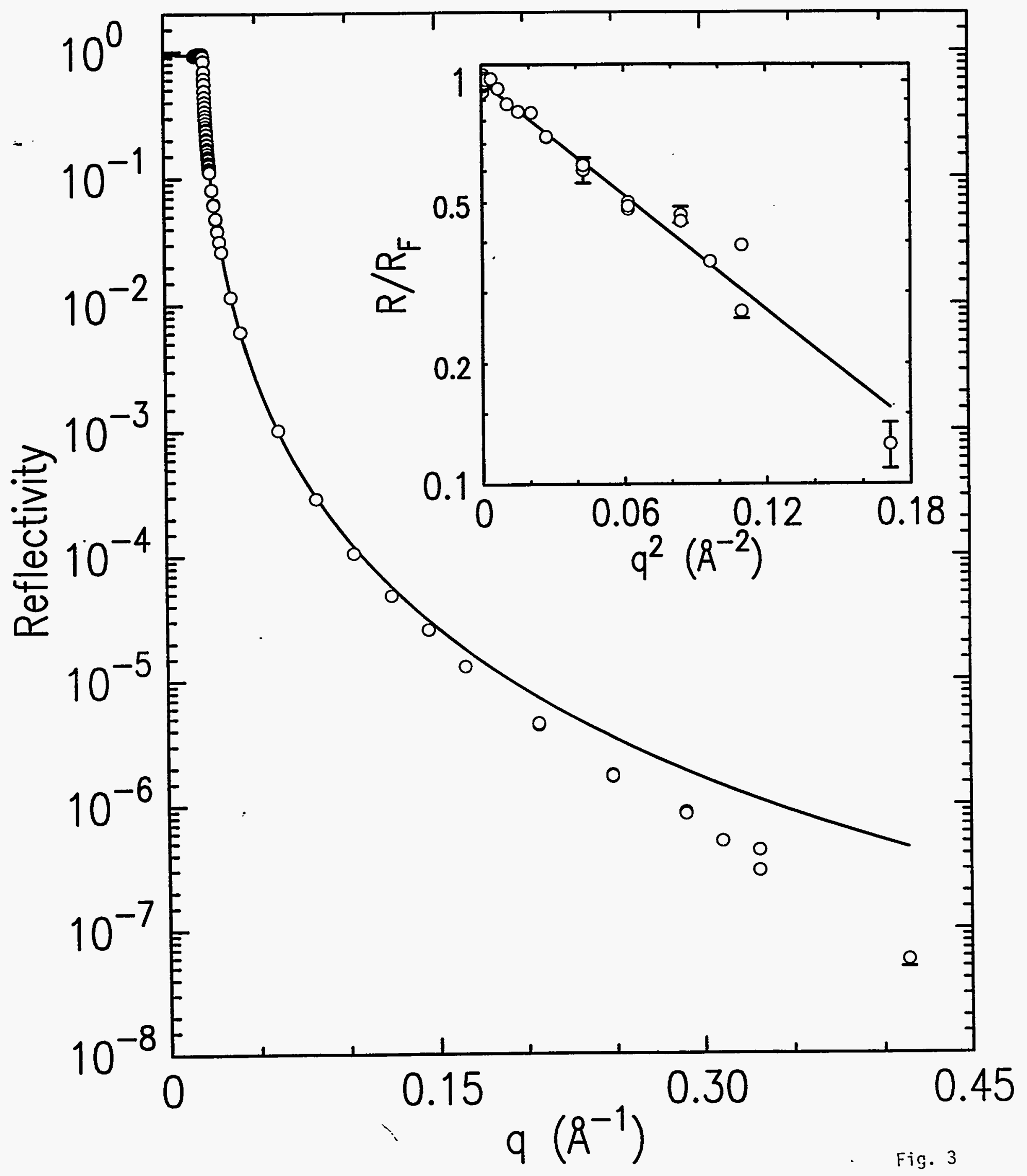




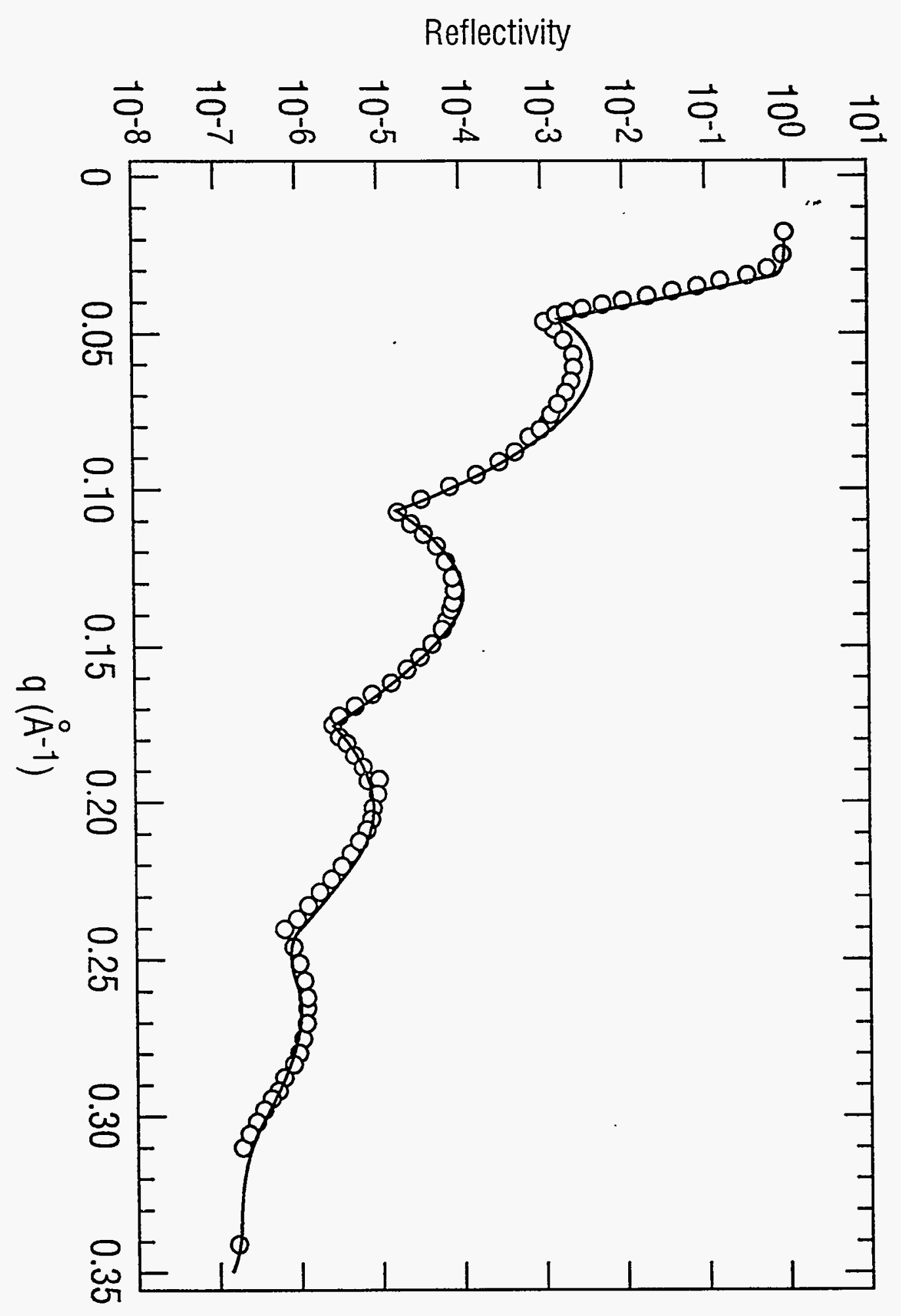




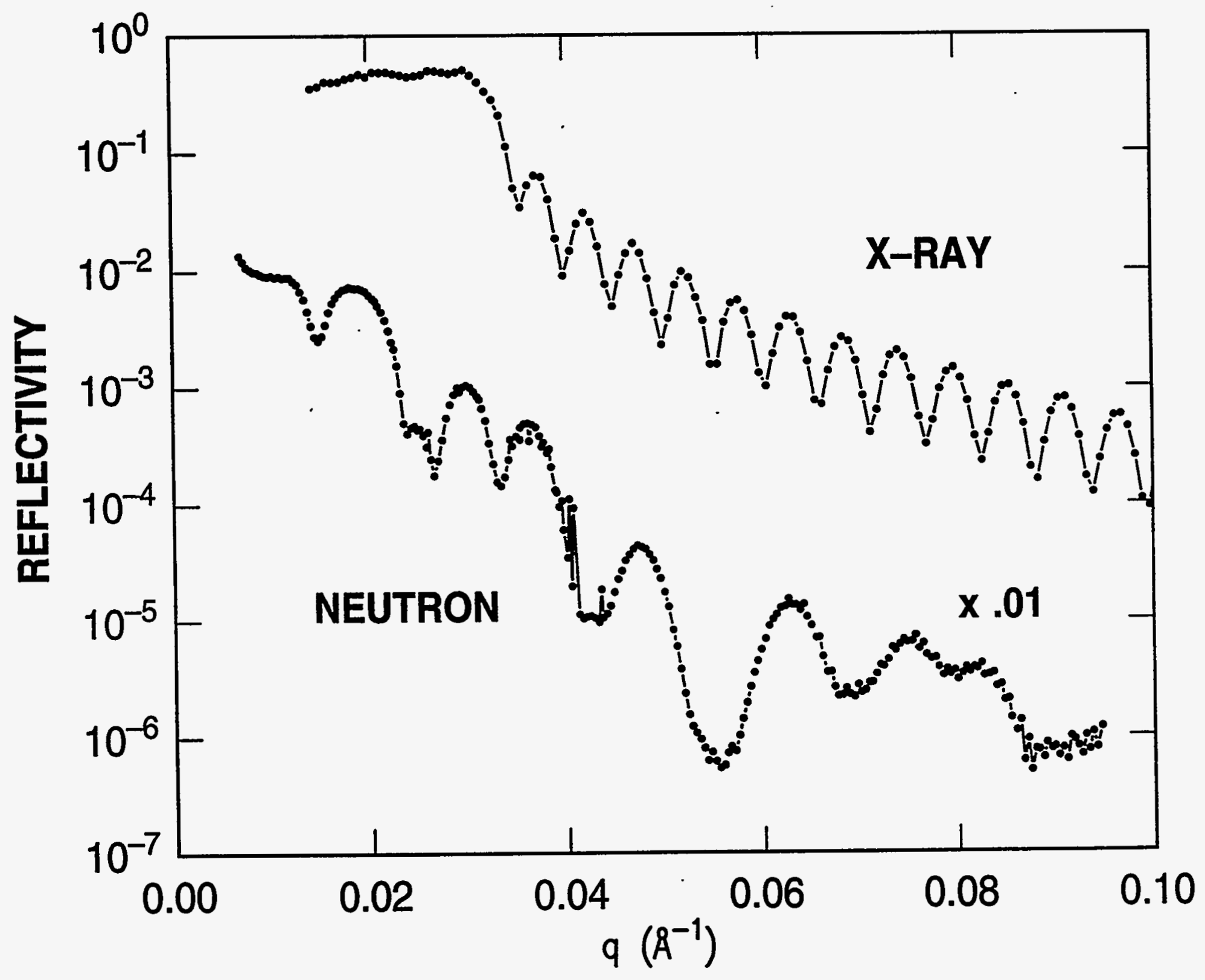

Fig. 5 


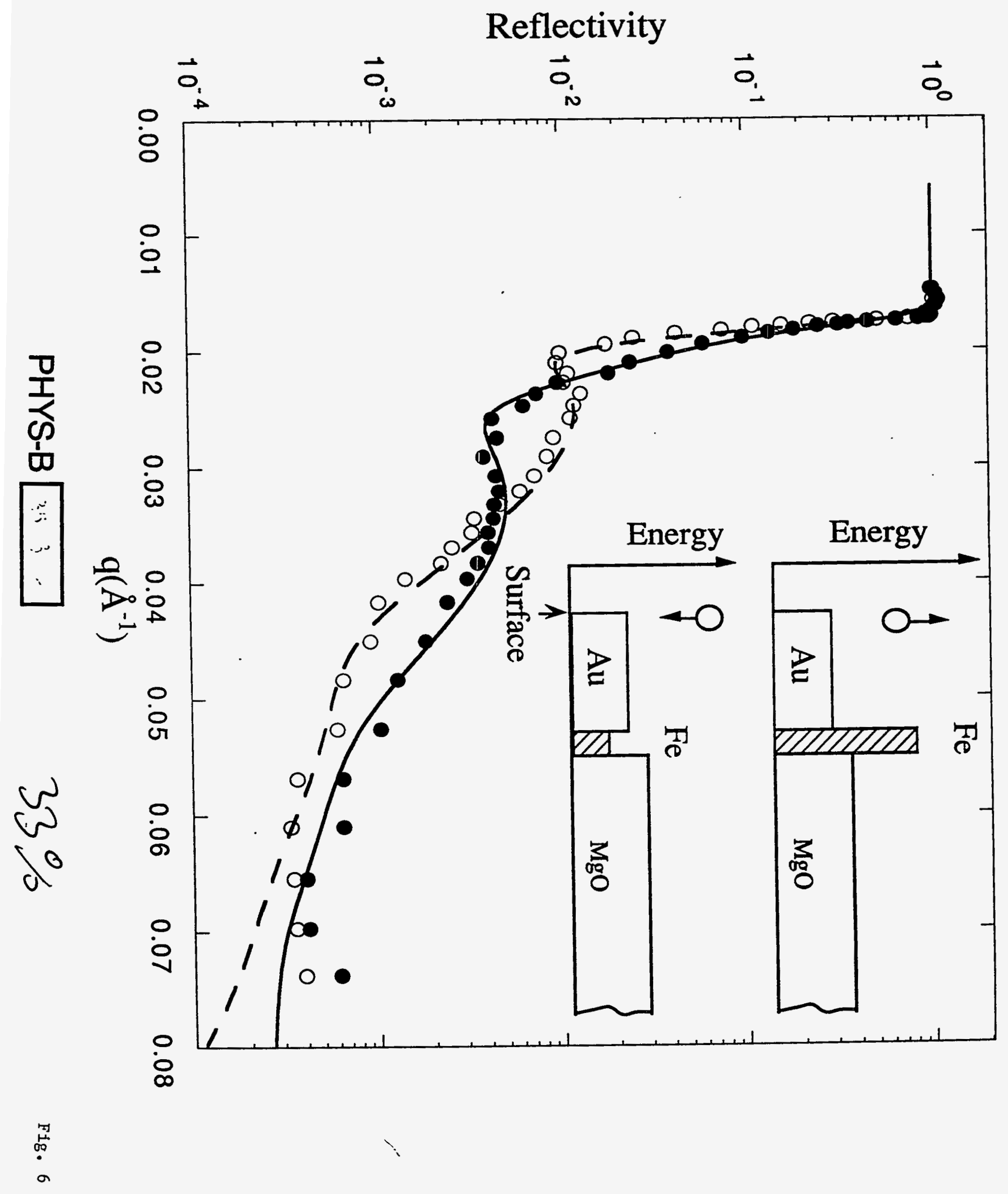




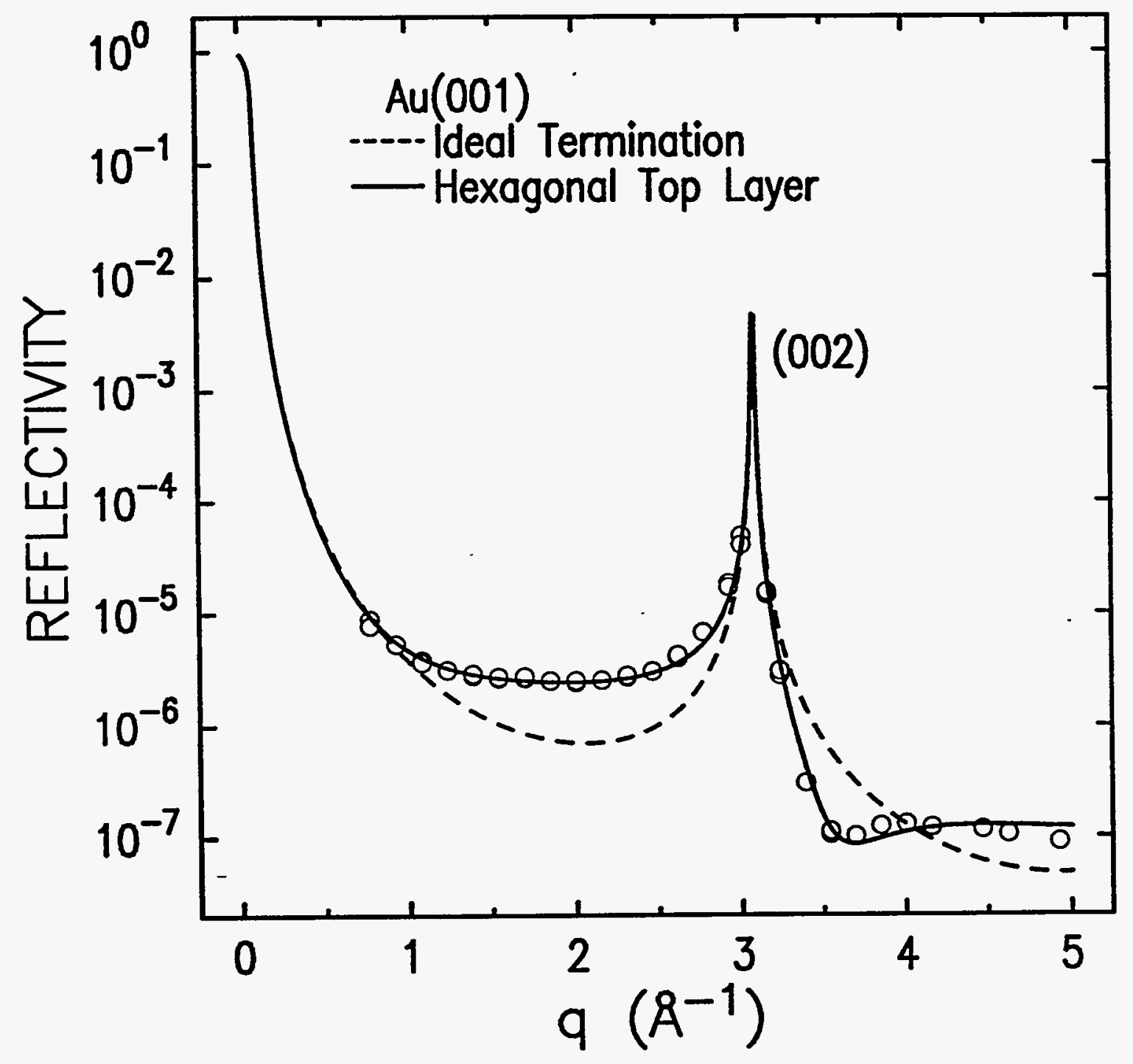

Fig。 7 